\title{
A COMPARISON BETWEEN INTERPOLATION METHODS FOR MORE ACCURATE ELEVATION SURFACE USING GNSS AND GIS
}

\author{
Farsat Heeto Abdulrahman ${ }^{1,}{ }^{*}$, Soleiman Foroughi ${ }^{*}$ and Sarhat Mustafa Adam ${ }^{*}$ \\ *Dept. of Surveying Engineering, College of Engineering, University of Duhok, \\ Kurdistan Region-Iraq \\ ${ }^{* *}$ College of Engineering, University of Duhok, Kurdistan Region-Iraq
}

(Accepted for Publication: December 8, 2020)

\begin{abstract}
Elevation surface is a fundamental element of spatial data that can be employed to perform a variety of geostatistical and spatial analyses. On this basis, this paper presents the assessment of elevation surfaces interpolation methods such as Inverse Distance Weighting (IDW), Ordinary kriging, and Local Polynomial Interpolation (LPI). There are three scenarios for achieving this purpose by examining it in three different areas: mild slope area, steep slope area and combined case. The dimension of each tested area is decided to be $100^{*} 100 \mathrm{~m}$ with 121 survey points for each. The ellipsoidal height of survey points are measured by the Global Navigation Satellite System (GNSS) receiver exploiting the real-time kinematic (RTK) technique. All survey points are transferred to Arc GIS environment for generating elevation surfaces and conducting interpolations. Interpolated pixels of surfaces are then compared with the trusted data collected with GNSS receiver in RTK mode. The result revealed that the total Root Mean Square (RMS) error for kriging interpolation in the steep area is around $26 \mathrm{~cm}$, whereas RMS for LPI interpolation in the flat area found to be approximately $8.0 \mathrm{~cm}$. The evaluation outcomes can be utilized to understand the influence of the slope on interpolation methods as well as to select the most appropriate method according to the ground relief.
\end{abstract}

KEYWORDS: Interpolation methods; IDW; Kriging, LPI; GPS; Geostatistical analysis.

\section{INTRODUCTION}

$\mathbf{T}$ The methods of visual representation of ground elevation are expanding subsequently. In modern times, the Digital Elevation Model (DEM), Digital Terrain Model (DTM) and Digital Surface Model (DSM) are databases of many spatial analyses such as 3D analysis and Raster Analysis in GIS. Surveying techniques are advancing rapidly such as using the drone and differential GPS as well as spatial interpolation methods in GIS environments. So far, a lot of research has been done on the comparison of interpolation methods.

(Arun \& Science, 2013), addressed that the Kriging method exhibited an improvement in its performance in comparison to other modern methods in most circumstances. The DEM obtained from SOI or SRTM data was not as suitable as the DEM generated from the DGPS data.

(Rishikeshan, Katiyar, \& Mahesh, 2014) stated that the Kriging method exhibited a medium range of accuracy and according to its performance which it comes next to natural neighbor, Topo to Raster and IDW. The DEM interpolated values have sensitivity to the interpolation method and one has to be extremely precise when selecting the interpolation method for the generation of DEM. In contrast, other studies (Aguilar, Agüera, Aguilar, Carvajal, \& Sensing, 2005; Arun \& Science, 2013; Brus et al., 1996; Declercq \& Systems, 1996; Gallichand \& Marcotte, 1993; Weber \& Englund, 1992) showed that IDW or RBFs (which were neighborhood strategies) produced results that were similar in accuracy to kriging as well as having the ability to increase in its precision.

On the other hand (Strößenreuther, Horwath, \& Schröder, 2020) investigated two approaches, satellite altimetry by repeat altimetry analysis (RAA) and different kinds of interpolation methods namely (ordinary kriging, IDW,filtered kriging and heterogeneous measurement-errorfiltered kriging) for surface elevation change in polar ice sheet. They found that the RAA have different findings based on the cell size and the

farsat.heeto@uod.ac; Soleiman.foroughi@gmail.com; sarhat@uod.ac

${ }^{1}$ Corresponding author: College of Engineering, University of Duhok, Kurdistan Region, Iraq. 
parameters of topography. The best result was found when the heterogeneous errors with HFK were involved. Also, the obtained height changes were improved by the filtering included in the FK and HFK algorithm.

This paper tried to eliminate the effect of spatial distribution and distance between sample points and select the best and most optimal methods of interpolation with the least amount of error in proportion to the topographic condition. The concepts and phrases used in this study are described below.

\subsection{Digital Elevation Model (DEM)}

DEM is a visual representation of elevation above specific datum which is displayed in three types namely, Contour Line, Triangular Irregular Network (TIN) and Grid (regular Squared cell), in which an elevation value is linked to each pixel. DEMs can be derived from LIDAR, surveying with drone, RTK, laser scanning, digitization of topographic map, photogrammetry techniques, radar interferometry, field surveys and etc.

Quality and precision level of DEM depends mainly on: pixel size and resolution, interpolation method and algorithm, as well as spatial distribution and density of sample point, terrain roughness and surveying techniques

\subsubsection{Spatial Interpolation}

Spatial Interpolation is the process of creating a raster surface with a regular network of cells in which each pixel has its own known value. In fact, spatial interpolation is calculating the unknown value of cells (pixel) with using known values of sample points.

The academic aspect of the literary work demonstrates several interpolation methods which are divided into global and local approaches. In the local approach, the value of an unidentified point based on the value of the neighborhood pixels is estimated. Prominent local methods found in literature include Inverse Distance Weighting (IDW), local polynomial, Nearest Neighbor (NN), and Radial Basis Functions (RBFs). In the global approach, all of the available sample points are used to generate estimations for a particular point according to certain interpolation methods such as polynomial interpolation functions (Burrough, 1998).

\subsubsection{Inverse Distance Weighted( IDW)}

Inverse distance weighted (IDW) interpolation is a local, exact and deterministic interpolation method. In this method, the distance between the unknown point and the known sample points around is the fundamental factor of the interpolation method. As the distance between the sampling points increases, their importance in interpolation decreases as expressed in 'Equation (1) (Achilleos, 2011).

$$
H_{P}=\frac{\sum_{i=1}^{n} h_{i} / d_{i}^{2}}{\sum_{i=1}^{n}\left[1 / d_{i}^{2}\right]}
$$

Where $\mathrm{Hp}$ is the calculated elevation of point $P$ where the interpolation is effected;

hi is the elevation used to calculate the height at the point.

$\mathrm{P}$; di is the distances of the points from point $\mathrm{P}$;

$\mathrm{n}$ is the number of points used in the interpolation procedure for estimating the elevation of point $\mathrm{P}$.

\subsubsection{Kriging}

Kriging is a geo statistical interpolation that uses semivariogram for discovering auto-correlation between sample points and for the fitted model. Semivariogram graphs out the variance of all pairs of data according to distance. Kriging is a multistep process; it includes exploratory statistical analysis of the data, variogram modeling and creating the surface. General kriging formula as expressed in 'Equation (2)' (ESRI, 2016 2018).

$$
\hat{Z}\left(S_{0}\right)=\sum_{i=1}^{N} \lambda_{i} Z\left(S_{i}\right)
$$

Where $\mathrm{Z}(\mathrm{si})$ is the measured value at the ith location

$\lambda i$ is an unknown weight for the measured value at the ith location, $\lambda \mathrm{i}$, depends on a fitted model to the measured points

$\mathrm{S}_{0}$ is the prediction location $\mathrm{N}$ is the number of measured values

\subsubsection{Local Polynomial Interpolation (LPI)}

Local polynomial Interpolation fits many polynomials for the interpolation of surface, each within a specified neighborhood that describes the number, size and shape of the neighborhoods. Local polynomial interpolation relies on the following assumptions (ESRI, 2016, 2018)

1. The samples were taken on a grid (where the samples are equally spaced).

2. The data values are normally distributed within the neighborhood that is searched.

farsat.heeto@uod.ac; Soleiman.foroughi@gmail.com; sarhat@uod.ac

${ }^{1}$ Corresponding author: College of Engineering, University of Duhok, Kurdistan Region, Iraq. 
1.2 Real Time Kinematics (RTK)

Real Time Kinematics (RTK) surveying mode is one of the Global Navigation Satellite System (GNSS) techniques that are based on carrier phase-based relative positioning. In this technique, no post processing is required .The expected positioning accuracy is of the order of $10 \mathrm{~mm}$ for the horizontal component and $20 \mathrm{~mm}$ for the vertical component. RTK mode uses two (or more) receivers (base and rover) by simultaneously tracking the same satellites. The base receiver remains stationary over the known point and the rover is moveable and carried in range pole or backpack. The base station is attached to a radio transmitter for the transmission of corrected coordinates in real time to rover receiver through the communication (radio) link.

\section{METHODOLOGY}

The main purpose of this article is to simply evaluate interpolation methods of topographic surfaces. It is additionally In other words, it aims to determine an optimal method of interpolation without considering the distance between the sample points and their spatial distribution. In order to achieve this purpose, high accuracy is required, mainly in centimeters. In this study, three topographic situations have been considered; flat ground, steep slope and combined case. Each of these sectors have dimensions that are equal to $100 * 100 \mathrm{~m}$ as well as 121 survey points. The spatial distribution of the survey points are regular and several checkpoints (CP) for each sector are selected randomly. Distance between survey points is set to $10 \mathrm{~m}$, as shown in Figure 1.

The procedures of the fieldwork are as follows: first, before the fieldwork, a regular grid of points of the three tested areas are prepared using the latest available orthoimages. The coordinates of the regular grid are transferred to Leica viva GS15 receiver. Employing RTK positioning mode, all of the points stored in GS15 must be prepared for stakeout on the ground. The GS10 receiver is fixed on a highly accurate point which works as a base station, more details about the accuracy of this point can be found on (Adam, 2017). The base station transmits correction in real-time to the rover receiver at each survey point through the communication (radio) link. Simultaneously with stake out, the coordinates of the points are measured again relative to GS10, the receiver at the base station. In addition to regular survey points, some checkpoints are also measured with the same positioning technique, these points are shown in Figure (1), left side. The measured points have been imported to Arc GIS Desktop10.6 then plotted to a Shape file. DEM is generated from measured survey points using different interpolation methods as mentioned before (Kriging, IDW, and LPI). Visual and statistical analyses have been adopted to evaluate the spatial interpolation method. Statistical analysis is performed by computing the deviations of interpolated height values from corresponding observed values in terms of root mean square error (RMSE).

Regarding the software steps of this research are as follows: The base point is processed in the Leica Infinity software environment. The survey points obtained through RTK surveying mode are added to Arc Map 10.6 software in DWG format. In order to exploit the survey points and perform spatial analysis, the points are converted to shape file format. Due to the Arc GIS is more compatible with this format. The next step, the geo-statistical analyst toolbar is used for visualize and explore normal distribution and autocorrelation of points. Semi- variogram, covariance cloud, Quantile-quantile (QQ) plot, are used in this step. Finally, the survey points are interpolated using different methods. The parameters used for interpolation are shown in the Table1. Then, the check points are used to determine the amount of error for each method. According to the results, the most suitable method of interpolation has been selected for each area and the total procedure is shown in Figure 


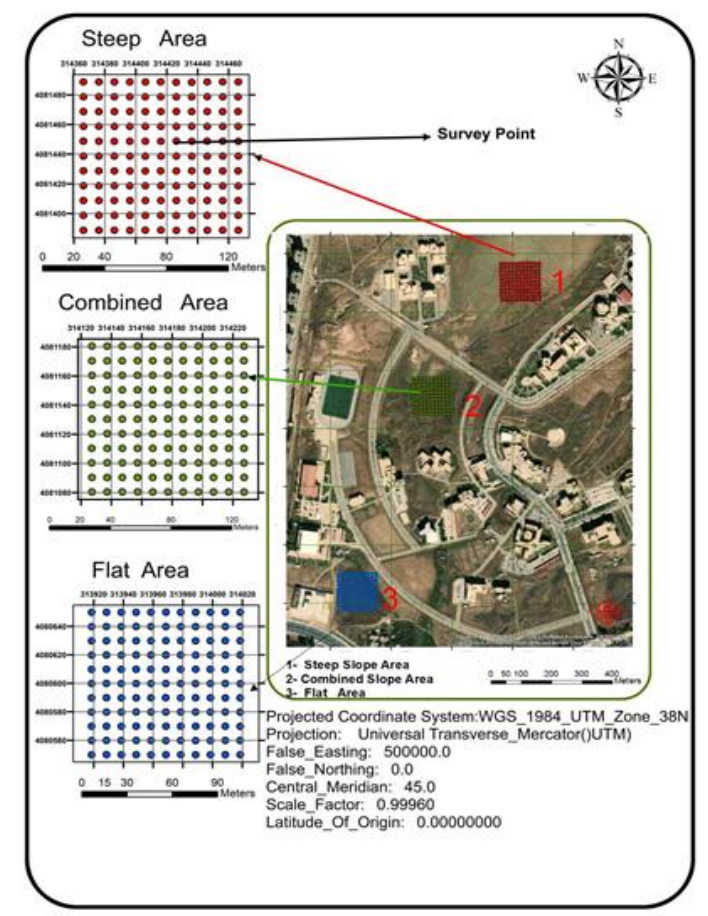

Fig. (1): Study area overlain grid of points of three sectors on bing satellite image

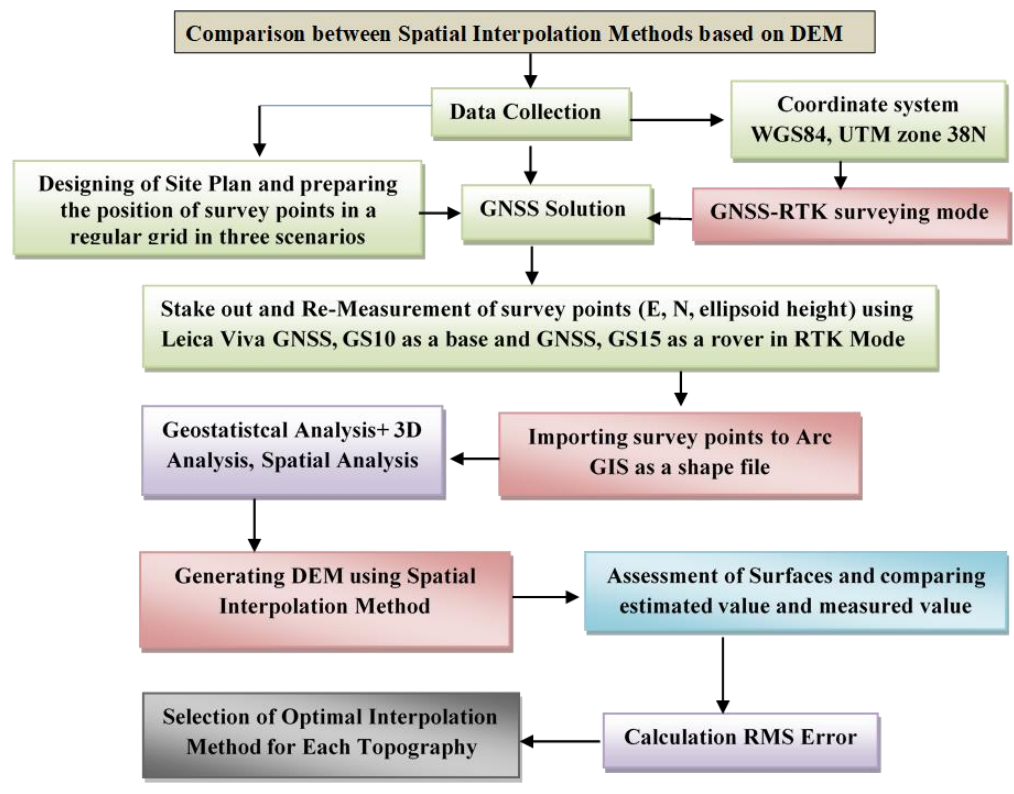

Fig. (2): Procedure of field work and office work

\section{RESULTS AND ANALYSIS}

The aim of this paper, is to evaluate the interpolation algorithms and check their accuracy and precision due to the topographic condition of the earth. To achieve this goal, three interpolated surfaces were prepared using 3 different methods: IDW, kriging and LPI (Map1, Map2 and Map3). The used interpolation parameters are shown in Table 1. To comprehend more coherently, the rate of the measured error value by RTK GPS surveying and the predicted value with interpolation methods is shown in Table 1. Total contributed points in each interpolating mode are 121 survey points, but three check points were displayed as a sample for each topographic situation as illustrated in Table2. The survey points Root mean Square Errors were calculated for final

farsat.heeto@uod.ac; Soleiman.foroughi@gmail.com; sarhat@uod.ac

${ }^{1}$ Corresponding author: College of Engineering, University of Duhok, Kurdistan Region, Iraq. 
Journal of University of Duhok, Vol. 23, No.2 (Pure and Eng. Sciences),Pp 342-351, 2020 (Special Issue)

$3^{\text {rd }}$ international conference on recent innovations in engineering (ICRIE) Duhok, September 9-10-2020

judgment about the interpolation method as depicted in $\quad$ Table

Table (1): Interpolation parameter for each method

\begin{tabular}{|c|c|c|c|}
\hline Interpolations Parameter & IDW & Kriging & LPI \\
\hline Maximum neighbors & 15 & 5 & 1000 \\
\hline Minimum neighbors & 10 & 2 & 10 \\
\hline Sector type & 1 sector & $\begin{array}{l}4 \text { Sectors with } 45^{\circ} \\
\text { offset }\end{array}$ & 1 Sector \\
\hline $\begin{array}{l}\text { Major and minor semi axis } \\
\text { (meter) }\end{array}$ & 35 & 141.620 & 160.212 \\
\hline
\end{tabular}

Table 2 shows that the predicted value for the same measured survey points vary according to the spatial interpolation method. The difference between predicted heights with each interpolation method with other methods is shown in Table 2. Also, the error rates of the predicted height is shown in a linear graph relative to the actual measured height with RTK GPS for each interpolation method and each topography situation. The predictive regression line is fitted in the linear graph (Refer to Map1, $2, \quad 3), \quad$ as shown in Figure 3.

farsat.heeto@uod.ac; Soleiman.foroughi@gmail.com; sarhat@uod.ac

${ }^{1}$ Corresponding author: College of Engineering, University of Duhok, Kurdistan Region, Iraq. 


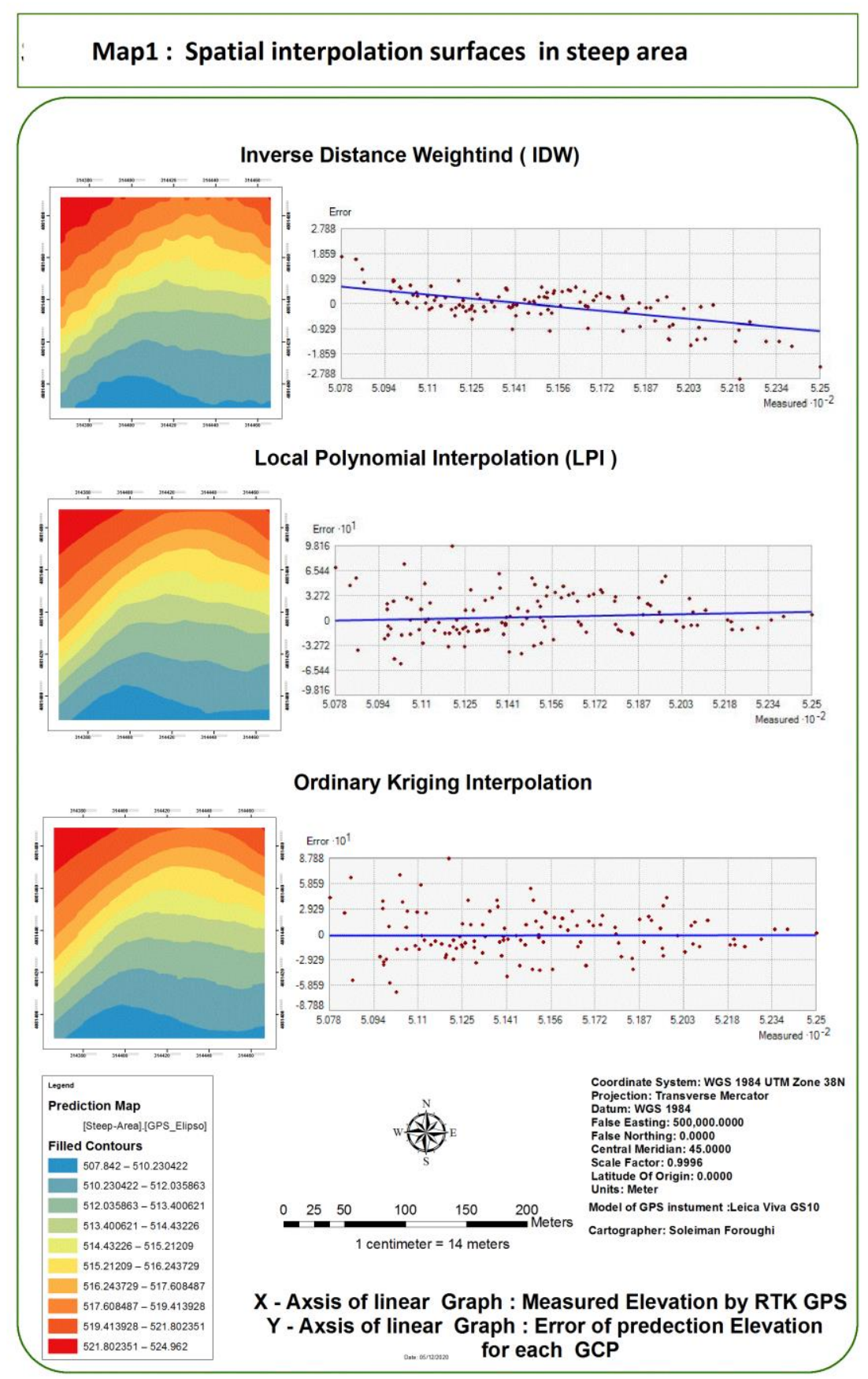

farsat.heeto@uod.ac; Soleiman.foroughi@gmail.com; sarhat@uod.ac ${ }^{1}$ Corresponding author: College of Engineering, University of Duhok, Kurdistan Region, Iraq. 


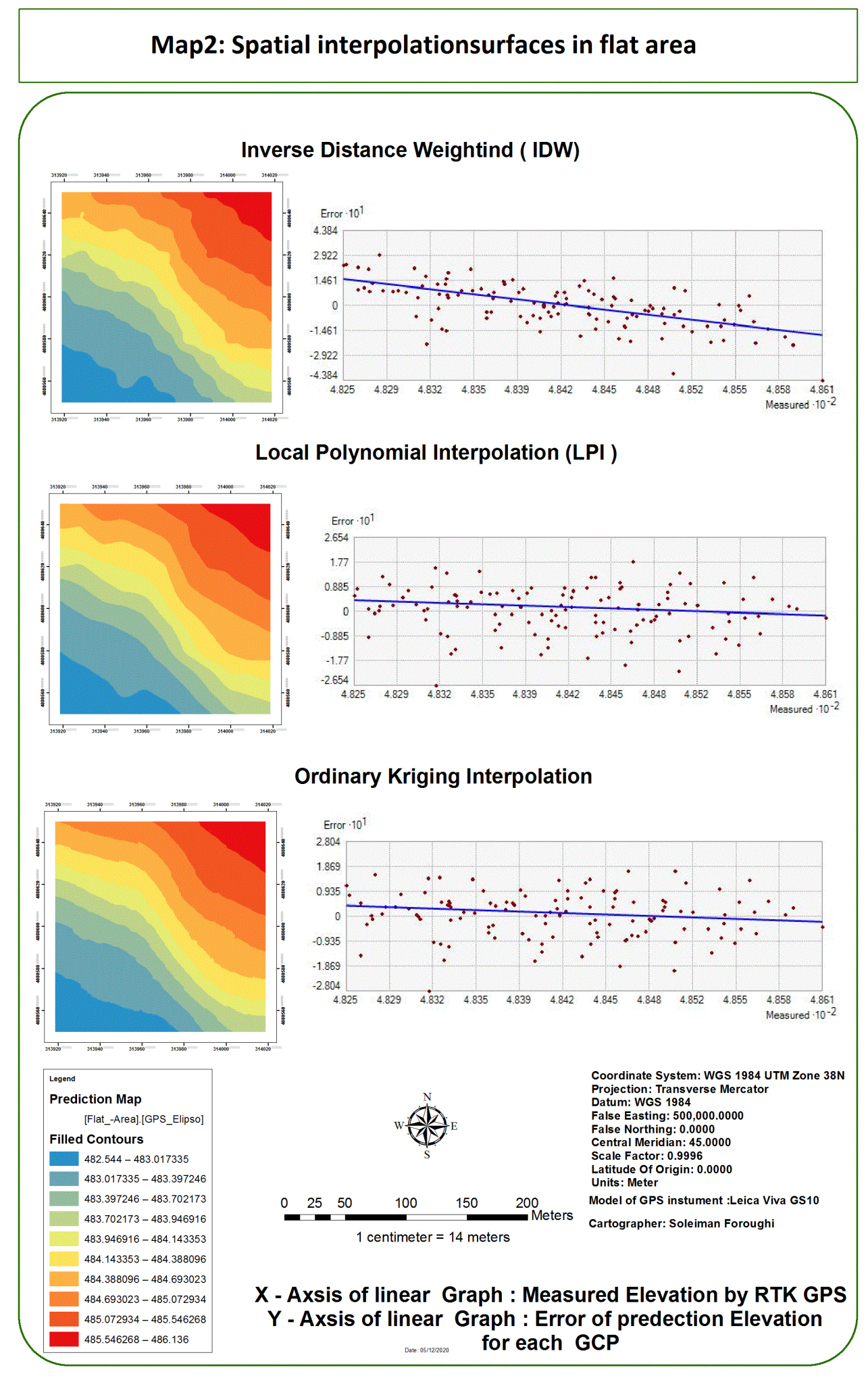

farsat.heeto@uod.ac; Soleiman.foroughi@gmail.com; sarhat@uod.ac 


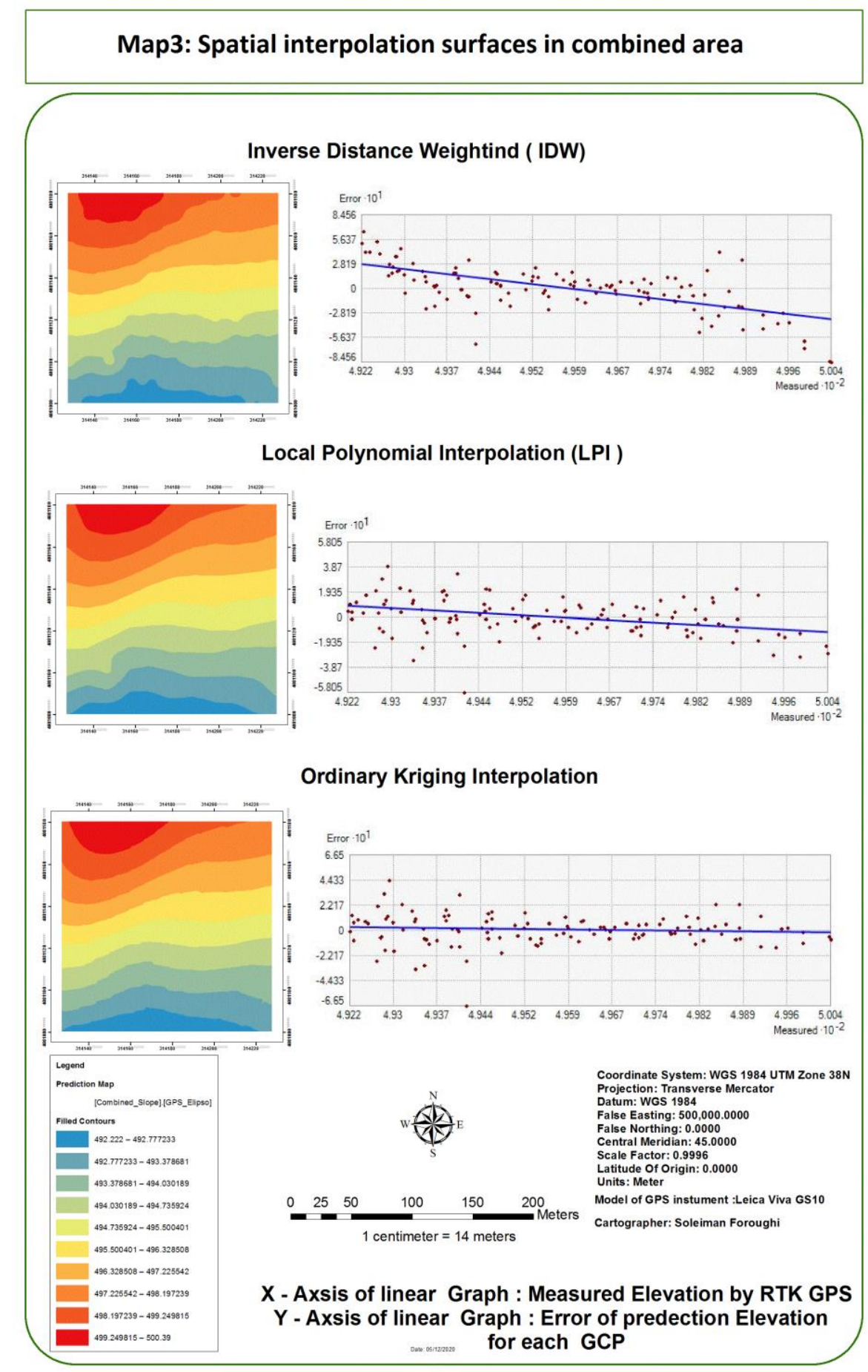

Fig. (3): Contour line color coded, and the predictive regression line is fitted in the linear graph. Map 1, interpolation surfaces in steep area. Map2, spatial interpolation in flat area. Map 3, spatial interpolation in combined area. In all three cases applied (IDW, LPI, and ordinary Kriging interpolation) methods.

farsat.heeto@uod.ac; Soleiman.foroughi@gmail.com; sarhat@uod.ac

${ }^{1}$ Corresponding author: College of Engineering, University of Duhok, Kurdistan Region, Iraq. 
Table (2): Ellipsoidal Height at check point from predicted by interpolation and RTK Observed Value in Meter Unit

\begin{tabular}{cccccc}
\hline Slope type & $\begin{array}{c}\text { Check } \\
\text { point ID }\end{array}$ & $\begin{array}{c}\text { RTK GPS } \\
\text { Measureme }\end{array}$ & \multicolumn{3}{c}{$\begin{array}{c}\text { Predicted Height with } \\
\text { Interpolation }\end{array}$} \\
\cline { 3 - 6 } & & nt & IDW & LPI & Kriging \\
\hline Steep Area & CP8 & 514.109 & 514.091 & 514.161 & 514.053 \\
\cline { 2 - 6 } & CP64 & 510.564 & 510.848 & 510.839 & 510.837 \\
\cline { 2 - 6 } & CP120 & 517.892 & 518.074 & 518.203 & 517.900 \\
\hline Combined & $\mathrm{CP5}$ & 493.478 & 493.508 & 493.541 & 493.496 \\
\cline { 2 - 6 } Area & $\mathrm{CP} 42$ & 495.079 & 495.064 & 495.050 & 495.028 \\
\cline { 2 - 6 } & $\mathrm{CP} 117$ & 495.996 & 495.844 & 495.889 & 495.910 \\
\hline Flat Area & $\mathrm{CP} 69$ & 484.043 & 484.039 & 484.047 & 484.047 \\
\cline { 2 - 6 } & $\mathrm{CP} 95$ & 485.022 & 484.969 & 484.919 & 484.925 \\
\cline { 2 - 6 } & $\mathrm{CP} 110$ & 483.666 & 483.709 & 483.535 & 483.584 \\
\hline
\end{tabular}

Table (3): Root Mean Square (RMS) errors (in meter unit)

\begin{tabular}{lccc}
\hline \multicolumn{1}{c}{ RMS } & $\begin{array}{c}\text { IDW } \\
\text { Interpolation }\end{array}$ & $\begin{array}{c}\text { LPI } \\
\text { Interpolation }\end{array}$ & $\begin{array}{c}\text { Kriging } \\
\text { interpolation }\end{array}$ \\
\hline Steep Area & 0.696 & 0.284 & 0.263 \\
\hline $\begin{array}{l}\text { Combined } \\
\text { Area }\end{array}$ & 0.266 & 0.145 & 0.135 \\
\hline Flat Area & 0.127 & 0.083 & 0.085 \\
\hline
\end{tabular}

Table 3 shows that the kriging interpolation method is more powerful in steep and combined area, and its flat area is $1.6 \%$ less than that of the LPI method. While in the other two areas (Steep and Combined Area), the LPI method is less powerful than kriging by $17 \%$ and $8 \%$ respectively. However, the IDW method has the lowest precision and maximum RMSE for all of the three tested areas. These findings revealed that creating DEM is found to be sensitive to height interpolation methods along with the nature of the terrain. (Arun \& Science, 2013) empahised that the Kriging interpolation method performs better when compared to other current methods in most environments.

\section{CONCLUSION}

The interpolated surfaces of ellipsoidal height vary according to the method of interpolation and its algorithm, nature of topography and distribution of ground sample point. Each spatial interpolation methods gives a different result. In this paper, among various methods of interpolation, only three methods were evaluated. The result showed that IDW has the lowest precision and the highest RMS error for all three scenarios among the interpolation methods.

By increasing the unevenness of the earth's slope, the capability of kriging method is revealed compared to other methods (RMS of kriging in steep area is equal to $0.262 \mathrm{~m}$ ). However, for flat land, the LPI method was found to have less RMS error (RMS of LPI interpolation in flat area is equal to $0.082 \mathrm{~m}$ ) (refer to Table 3).

\section{REFERENCES}

Achilleos, G. (2011). The Inverse Distance Weighted interpolation method and error propagation mechanism-creating a DEM from an analogue topographical map. Journal of Spatial Science, 56(2), 283-304.

Adam, S. M. (2017). THE USE OF ONLINE AND OFFLINE PROCESSING TOOLS TO IMPROVE THE PRECISION OF A GPS PASSIVE STATION. Journal of Duhok University, 20(1), 335-346. doi: 10.26682/sjuod.2017.20.1.30

Aguilar, F. J., Agüera, F., Aguilar, M. A., Carvajal, F. J. P. E., \& Sensing, R. (2005). Effects of terrain morphology, sampling density, and interpolation methods on grid DEM accuracy. 71(7), 805-816.

Arun, P. V. J. T. E. J. o. R. S., \& Science, S. (2013). A

farsat.heeto@uod.ac; Soleiman.foroughi@gmail.com; sarhat@uod.ac

${ }^{1}$ Corresponding author: College of Engineering, University of Duhok, Kurdistan Region, Iraq. 
comparative analysis of different DEM interpolation methods. 16(2), 133-139.

Brus, D., De Gruijter, J., Marsman, B., Visschers, R., Bregt, A., Breeuwsma, A., \& Bouma, J. J. E. (1996). The performance of spatial interpolation methods and choropleth maps to estimate properties at points: a soil survey case study. 7(1), 1-16.

Burrough, P. A. (1998). Principles of geographical information systems.

Declercq, F. A. N. J. C., \& Systems, G. I. (1996). Interpolation methods for scattered sample data: accuracy, spatial patterns, processing time. 23(3), 128-144.

ESRI. (2016). How local polynomial interpolation works. Retrieved 16 April, 2020, from https://desktop.arcgis.com/es/arcmap/10.4/exte nsions/geostatistical-analyst/how-localpolynomial-interpolation-works.htm

ESRI. (2016 ). How Kriging works. Retrieved 16 April, 2020, from https://desktop.arcgis.com/en/arcmap/10.4/tool s/3d-analyst-toolbox/how-kriging-works.htm

ESRI. (2018). geostatistical-analyst. Retrieved 16
April, 2020 from https://desktop.arcgis.com/es/arcmap/10.4/exte nsions/geostatistical-analyst.htm

Gallichand, J., \& Marcotte, D. J. G. (1993). Mapping clay content for subsurface drainage in the Nile Delta. 58(3-4), 165-179.

Rishikeshan, C., Katiyar, S., \& Mahesh, V. V. (2014). Detailed evaluation of DEM interpolation methods in GIS using DGPS data. Paper presented at the 2014 International Conference on Computational Intelligence and Communication Networks.

Strößenreuther, U., Horwath, M., \& Schröder, L. (2020). How Different Analysis and Interpolation Methods Affect the Accuracy of Ice Surface Elevation Changes Inferred from Satellite Altimetry. Mathematical Geosciences, 1-27.

Weber, D., \& Englund, E. J. M. G. (1992). Evaluation and comparison of spatial interpolators. 24(4), 381-391.

farsat.heeto@uod.ac; Soleiman.foroughi@gmail.com; sarhat@uod.ac

${ }^{1}$ Corresponding author: College of Engineering, University of Duhok, Kurdistan Region, Iraq. 